\title{
Surgical Therapy of Sporadic Pancreatic Neuroendocrine Neoplasias G1/G2
}

\author{
Volker Fendrich ${ }^{a}, \mathrm{~b} \quad$ Detlef K. Bartsch ${ }^{b}$ \\ ${ }^{a}$ Department of Endocrine Surgery, Schön Klinik Hamburg, Hamburg, Germany; \\ ${ }^{b}$ Department of Visceral-, Thoracic- and Vascular Surgery, University Hospital Marburg, Marburg, Germany
}

\section{Keywords}

Neuroendocrine tumors of the pancreas - Insulinoma . Gastrinoma - Non-functional pancreatic tumors . Liver metastases · Surgical therapy

\section{Summary}

Background: Pancreatic neuroendocrine neoplasias (pNENs) are uncommon but fascinating tumors with an annual incidence of 1 per 100,000 people. pNENs present either as functional tumors, causing specific hormonal syndromes like Zollinger-Ellison syndrome (ZES) or organic hyperinsulinism, or as non-functional pancreatic tumors (NF-pNENs). The natural history of pNENs is highly variable. $90 \%$ of all insulinomas or small NFpNENs are readily curable by surgical resection. Most other functional and late detected NF-pNENs have a less favorable chance for cure. Methods: A systematic review of the literature was performed to identify the current state of the art with regard to the key issues of surgery in pNEN G1/G2. Results: This article provides a comprehensive review of the current literature addressing the current challenges in pNEN surgery. Conclusion: Patients with completely resected tumors generally have a good prognosis, and an aggressive surgical approach combined with conservative treatment options in patients with advanced disease rarely provides cure but often results in long-term survival.

(C) 2017 S. Karger GmbH, Freiburg

\section{Introduction}

Pancreatic neuroendocrine neoplasias (pNENs) represent an important subset of pancreatic neoplasms (table 1). They account for $2-4 \%$ of all clinically detected pancreatic tumors. pNENs are rare tumors, but are detected more and more often $[1,2]$. pNENs show no significant gender predilection and occur at all ages. They consist of single or multiple neoplasias and are associated in 10-20\% with multiple endocrine neoplasia type 1 (MEN1). pNENs present either as functional tumors, causing specific hormonal syndromes like Zollinger-Ellison syndrome (ZES) or organic hyperinsulinism, or as non-functional pNENs (NF-pNENs) with symptoms similar to pancreatic adenocarcinoma [1]. This article focuses on the surgical management of sporadic pNENs G1/G2 and their liver metastases (LM).

\section{Short Overview of Clinical Characteristics of Pancreatic Neuroendocrine Neoplasias}

\section{Insulinomas}

Insulinomas are the most frequent of all functioning pNENs. Virtually all insulinomas are located in the pancreas. Approximately $90 \%$ of insulinomas are solitary; the remaining $10 \%$ are multiple and are associated with MEN1 syndrome [3]. Most insulinomas are small. $40 \%$ are less than $1 \mathrm{~cm}, 66 \%$ are less than $1.5 \mathrm{~cm}$, and $90 \%$ are less than $2 \mathrm{~cm}$ in diameter. Only $10 \%$ of the tumors are malignant at the time of diagnosis.

Insulinomas are characterized by fasting hypoglycemia and neuroglycopenic symptoms, and occasionally sympathoadrenal autonomic symptoms [3]. Whipple developed a symptom triad bearing his name to identify patients with insulinoma more accurately. These symptoms include signs and symptoms of hypoglycemia after fasting or exercise, blood glucose of less than $45 \mathrm{mg} / \mathrm{dl}$ when symptomatic, and symptoms relieved by intravenous or oral glu-

\section{KARGER}

(c) 2017 S. Karger GmbH, Freiburg

Fax +497614520714
Prof. Dr. med. Detlef K. Bartsch

Klinik für Visceral-, Thorax- und Gefäßchirurgie

Universitätsklinikum Marburg

Baldingerstraße, 35043 Marburg, Germany

bartsch@med.uni-marburg.de 
Table 1. Neuroendocrine neoplasias of the pancreas

\begin{tabular}{|c|c|c|}
\hline Tumor (syndrome) & Incidence, \% & Presentation \\
\hline Insulinoma & $70-80$ & $\begin{array}{l}\text { weakness, sweating, tremulousness, tachycardia, anxiety, fatigue, headache, dizziness, } \\
\text { disorientation, seizures, and unconsciousness }\end{array}$ \\
\hline Gastrinoma & $20-25$ & $\begin{array}{l}\text { intractable or recurrent peptic ulcer disease (hemorrhage, perforation), complications } \\
\text { of peptic ulcer, diarrhea }\end{array}$ \\
\hline VIPoma & 4 & profuse watery diarrhea, hypotension, abdominal pain \\
\hline Glucagonoma & 4 & $\begin{array}{l}\text { migratory, necrolytic skin rash, glossitis, stomatitis, angular cheilitis, diabetes, } \\
\text { severe weight loss, diarrhea }\end{array}$ \\
\hline Somatostatinoma & $<5$ & weight loss, cholelithiasis, diarrhea, neurofibromatosis \\
\hline Carcinoid & $<1$ & flushing, sweating, diarrhea, edema, wheezing \\
\hline ACTHoma & $<1$ & Cushing's syndrome \\
\hline GRFoma & $<1$ & acromegaly \\
\hline PTH-like-oma & $<1$ & hypercalcemia, bone pain \\
\hline Neurotensinoma & $<1$ & hypotension, tachycardia, malabsorption \\
\hline Non-functional tumors & $30-50$ & obstructive jaundice, pancreatitis, epigastric pain, duodenal obstruction, weight loss, fatigue \\
\hline
\end{tabular}

cose. These symptoms usually occur when serum glucose is less than $40 \mathrm{mg} / \mathrm{dl}$ [4]. A fasting test that may last up to $72 \mathrm{~h}$ is regarded as the most sensitive test. About $80 \%$ of insulinomas are diagnosed by this test, most of them in the first $24 \mathrm{~h}$ [5].

\section{Non-Functioning pNENs}

Clinically, NF-pNENs produce no or insufficient quantities of hormones, such as pancreatic polypeptide, that do not cause any hormonal symptoms [6]. Because of modern imaging modalities, they have been diagnosed more frequently and now represent at least $50 \%$ of pNENs. At operation these tumors are generally larger than their functional counterparts and are equally located throughout the pancreas. Patients usually present late owing to the lack of a clinical/hormonal marker of the tumor's activity. Therefore, in contrast to functioning pNENs, patients with NF-pNENs present with either various non-specific symptoms, such as abdominal pain and weight loss, or pancreatitis. Nowadays, however, more and more asymptomatic NF-pNENs are detected incidentally during an imaging procedure for other reasons.

Measurement of detectable serum or plasma levels of various hormones can establish the diagnosis of an NF-pNEN. Chromogranin $\mathrm{A}(\mathrm{CgA})$ is considered the best tumor marker currently available for the evaluation and follow-up of patients with NFpNENs, as these tumors do not reliably produce any other suitable marker. Plasma CgA is elevated in $60-100 \%$ of patients with NFpNENs. Furthermore, up to $75 \%$ of NF-pNENs are associated with increased serum levels of pancreatic polypeptide [6].

\section{Gastrinomas (Zollinger-Ellison Syndrome)}

Gastrinomas were first described in 1955 when Zollinger and Ellison, of the Ohio State University Medical School, reported on
2 patients with islet cell tumors associated with atypical peptic ulceration of the jejunum [7]. Approximately $0.1 \%$ of patients with duodenal ulcers show evidence of ZES. The reported incidence is between 0.5 and 4/1,000,000/year. The anatomical area harboring the vast majority of these tumors comprise the head of the pancreas, the superior and descending portion of the duodenum, and the relevant lymph nodes and has been termed the 'gastrinoma triangle' [8].

In patients with ZES abdominal pain is the most frequent complaint either alone or with diarrhea, followed by heartburn, nausea, or bleeding. The abdominal pain is primarily due to peptic ulcer disease or gastroesophageal reflux disease and is indistinguishable in character from that seen in ordinary ulcer patients [9].

If the patient presents a gastric $\mathrm{pH}$ below 4.0 and a serum gastrin concentration above $1,000 \mathrm{pg} / \mathrm{ml}$ (normal $<100 \mathrm{pg} / \mathrm{ml}$ ), then the diagnosis of ZES is confirmed.

\section{Rare Functioning pNENs}

\section{Vipomas}

Vasointestinal peptide-secreting tumors, also called VIPomas, Verner-Morrison syndrome, or WDHA (watery diarrhea, hypokalemia, and acidosis), account for fewer than $5 \%$ of islet cell tumors [1]. The 2 patients described by Verner and Morrison died from dehydration and renal failure in spite of attempted intravenous hydration. The VIP directly inhibits gastric acid secretion, causing achlorhydria. Sporadic VIPomas are solitary tumors and arise from the VIP-secreting cells that are usually located in the region of the pancreatic tail and body [1]. More than $90 \%$ of these tumors are $<2$ $\mathrm{cm}$ in size as well as malignant and metastasize to the lymph nodes, liver, and bone. The secretory diarrhea ranges between 0.5 and 15 $1 / 24 \mathrm{~h}$ and is usually the most prominent symptom at presentation. It results in severe loss of potassium and bicarbonate, which in turn leads to metabolic acidosis and dehydration. The 5-year survival 
rate is $60 \%$ for patients with metastases and over $90 \%$ for patients without distant metastases [1].

\section{Glucagonomas}

Glucagonomas arise from the glucagon-producing $\alpha$-cells of the pancreas. Around $60 \%$ of patients already have LM at the time of diagnosis [1]. Tumors that produce excessive glucagon cause a specific syndrome of diabetes mellitus, a skin rash (necrolytic migratory erythema), hypoaminoacidemia, and a tendency for deep venous thrombosis. Patients often also have stomatitis, glossitis, and cheilosis associated with the skin rash. The syndrome is diagnosed by elevated plasma levels of glucagon. Levels greater than 1,000 pg/ $\mathrm{ml}$ are diagnostic of the syndrome. Once the syndrome is diagnosed, surgical resection of the tumor is indicated whenever possible. Preoperatively a management with somatostatin analogs and nutritional supplementation is indicated to correct the nutritional deficiency and to resolve the rash [10].

\section{Current Surgical Treatment Strategies for Sporadic Pancreatic Neuroendocrine Neoplasias}

\section{Insulinoma}

Surgical cure rates in patients with the biochemical diagnosis of insulinoma range from 77 to $100 \%$ [3]. At surgical exploration, the abdomen is initially explored for evidence of metastatic disease. Then a meticulous surgical exploration, i.e. an extended Kocher maneuver to be able to palpate the head, as well as the mobilization of the distal pancreas and the spleen should follow to explore the body and tail of the gland to examine the distal pancreas carefully and completely. Intraoperative ultrasonography (IOUS) should then be used to confirm the presence of the insulinoma or to detect non-palpable lesions and also to discern the relation of the tumor to the pancreatic duct. Identification of the pancreatic duct and determination of its proximity to the insulinoma can guide safe enucleation of the tumor. This approach can minimize the likelihood of a postoperative pancreatic fistula. Tumor enucleation, when feasible, is the technique of choice. If the tumor is located in the pancreatic tail, a distal spleen-preserving pancreatic resection might be the procedure of choice. In order to be considered malignant, these tumors must show evidence of either local invasion into surrounding soft tissue or verification of lymph node or LM. Malignant insulinomas account for only about $5-10 \%$ of all insulinomas. Aggressive attempts for resection are indicated, since there is no effective medical treatment option to control hypoglycemia. Malignant insulinomas located in the body or tail of the pancreas are effectively treated by distal pancreatectomy with splenectomy and lymphadenectomy. For tumors located in the head of the gland, resection requires partial pancreaticoduodenectomy (PPD) [11].

Recent advances in laparoscopic technique and instrumentation have enabled surgeons to approach complex procedures laparoscopically. This is also true for insulinomas [12-14]. The patient is placed in a half-lateral position with the left side up for tumors lo-

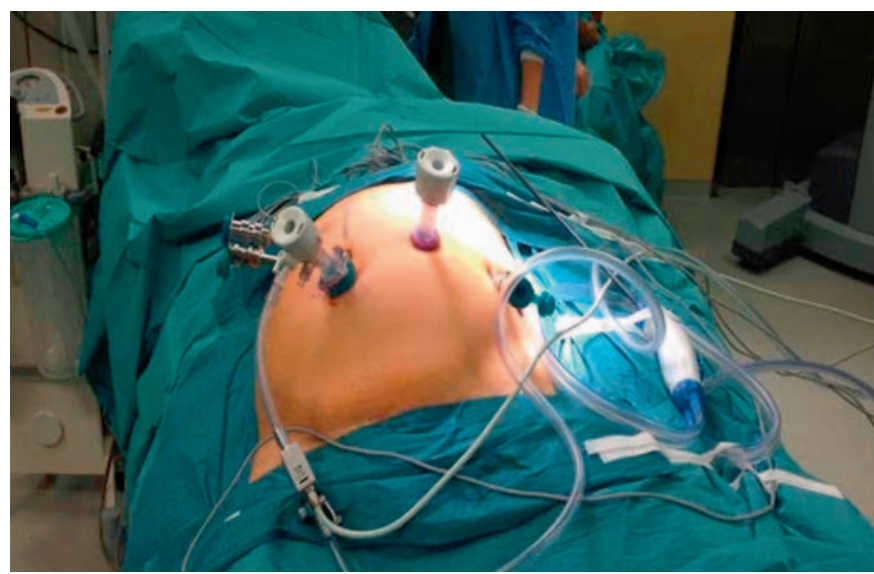

Fig. 1. Laparoscopic operation for pancreatic neuroendocrine neoplasias. The patient is placed in a half-lateral decubitus position with the left side uppermost for tumors in the body/tail of the pancreas. The surgeon and the assistant stand on the left of the patient, while the cameraman and the scrub nurse are on the opposite side. Two monitors are used. Typical port sites for resection of lesions in the body/tail of the pancreas are shown.

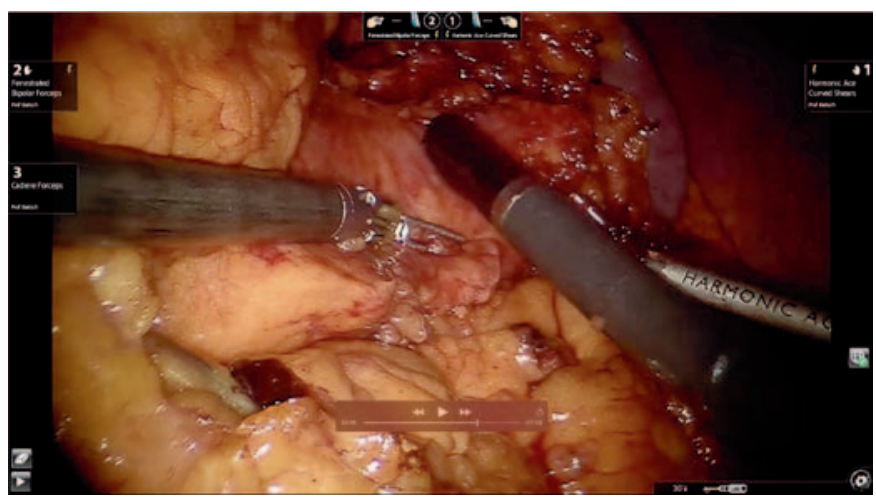

Fig. 2. Laparoscopic ultrasound.

cated in the body or tail of the pancreas or with the right side up for tumors in the head of the gland, and in the reverse Trendelenburg position. Four 10-12 mm trocars are inserted in the abdominal wall: 3-4 cm above the umbilicus, in the xiphoid area, subcostal on the midaxillary line, and in the subcostal midclavicular line (fig. 1). The pancreas is exposed after opening the lesser sac after mobilizing its head. Laparoscopic ultrasound should be used to identify non-visible tumors and to determine the relationship of the lesion to surrounding veins and the pancreatic duct (fig. 2). Laparoscopic ultrasound can be particularly helpful in identifying lesions in the tail that are often missed by endoscopic ultrasound. For superficial ventral tumors, laparoscopic enucleation is undertaken with electrocautery or laparoscopic vessel sealing shears. Small pancreatic vessels can be clipped and cut. Tumors located deep in the body or tail of the pancreas and those in close proximity to the pancreatic duct ( $<3 \mathrm{~mm}$ distance) require distal pancreatectomy. In cases where visualization and ultrasound fail, a hand port can be used to allow palpation of the gland. Tumors situated very distally near the splenic hilum are especially difficult to identify. It is worthwhile preserving the spleen during this procedure, if 
it can be safely accomplished. The pancreatic tail and/or body should be meticulously dissected from the splenic vessels, or these vessels may be resected together with the pancreas, leaving the spleen vascularized by the short gastric vessels [15].

Postoperatively, blood sugar levels begin to rise in most patients within the first hours after the removal of an insulinoma (reactive hyperglycemia). To preserve pancreatic function and to reduce the risk of iatrogenic diabetes mellitus, patients in whom tumor identification is not successful at operation should not undergo blind resection.

\section{Non-Functioning pNENs}

According to the classification of the World Health Organization, the size of the endocrine tumor correlates with malignant growth. Therefore, in localized tumors larger than $2 \mathrm{~cm}$, aggressive surgery and, if required, resection of adjacent organs (stomach, colon, kidney, adrenal gland) and/or major venous resection is indicated [16]. At the present time, most would advocate an aggressive surgical approach for the management of malignant NFpNENs even in the presence of localized metastases [11, 16]. The major goal is a potentially curative $\mathrm{R} 0$ resection by either partial pancreatoduodenectomy or distal splenopancreatectomy depending on the localization of the tumor. As lymph node metastases are frequently encountered, regional lymphadenectomy with en bloc resection of the primary tumor is the goal $[11,16]$. In contrast, only few data exist with respect to a positive effect of surgery on overall survival in small $(<2 \mathrm{~cm})$, possibly benign or intermediate-risk pancreatic endocrine tumors. In a very recent study, Partelli et al. [17] analyzed five retrospective studies with a total of 540 patients. Of these, 327 patients underwent active surveillance and $213 \mathrm{had}$ surgery. The median length of follow-up ranged from 28 to 45 months. Measurable tumor growth was observed in $0-51 \%$ of patients. Overall, 46 patients (14\%) underwent pancreatic resection after initial conservative treatment. In most patients the reason was an increase in tumor size (19 of 46). There were no disease-related deaths in the active surveillance group in any of the studies. This systematic review suggests that active surveillance of patients affected by sporadic, small, asymptomatic NF-pNENs may be a good alternative to surgical treatment. There are some criteria to opt for surgery even if the tumor is $<2 \mathrm{~cm}$ : the wish of the patient to end a lifelong surveillance, a progression of $20 \%$ of the tumor in 12 months, or suspicious lymph nodes. However, the possibility of surgical cure has to be weighed against the operative morbidity, mortality, and long-term complications associated with pancreatic surgery [16].

\section{Laparoscopic and Robotic Surgery for Insulinomas and Non-Functioning pNENs}

As already mentioned, most patients with insulinomas are ideal candidates for a minimally invasive approach, because these tu- mors are small, solitary, and benign. The first successful laparoscopic resection was reported by Gagner in 1996 [18]. As mentioned earlier, the most sensitive method of localization is intraoperative palpation and IOUS. In laparoscopic surgery, palpation is not possible. We and others have reported that preoperative localization, mainly by endoscopic ultrasonography, is crucial for the decision to operate laparoscopically $[19,20]$ and that minimally invasive surgery for pNENs should be undertaken only if laparoscopic ultrasound is available. Laparoscopic ultrasound helps the surgeon to decide whether to use enucleation or resection, a decision that will depend on the proximity to the main pancreatic duct or large blood vessels.

Laparoscopic enucleation is reserved for tumors less than $2 \mathrm{~cm}$ in diameter located on, or near, the surface of the pancreas, and not in contact with splenic vessels, the portal vein, or the main pancreatic duct. If these criteria are not met, laparoscopic spleen-preserving distal pancreatectomy should be the preferred choice. Recent small-scale retrospective studies have shown that the rate of spleen and vessel preservation is higher with the robotic approach than with the conventional laparoscopic approach for distal resections $[21,22]$.

Gastrinomas are not yet considered as candidates for a laparoscopic approach for two reasons. First, most gastrinomas are usually located in the duodenum, and bidigital palpation after duodenotomy (DUODX) is essential to identify the small tumor. Second, most pancreatic gastrinomas are over $2 \mathrm{~cm}$ in diameter at diagnosis and reveal metastases in up to $70 \%$ of patients, thus requiring a meticulous lymphadenectomy, which is not ideal for a laparoscopic approach. The same holds true for rare functioning pNENs [11].

Given the current data, minimally invasive enucleation or resection of insulinoma and most NF-pNENs is feasible and safe so that it is considered the procedure of choice for selected insulinomas [1, 12-14]. Nevertheless, it seems clear that such a treatment should only be offered by surgeons who are experienced in both endocrine pancreatic operations and advanced laparoscopic surgery. The same is true for robotic surgery, which emerged in the last years as a new technical possibility $[22,23]$. As technology advances and experience with robotic-assisted surgery will increase, it is likely to become an alternative method of pancreatic resection for pNENs (fig. 3, 4).

\section{Gastrinoma}

As with all pNENs, the only chance for cure of gastrinoma is complete surgical resection, which is achieved in $26-100 \%$ of patients. A study compared 160 patients with ZES undergoing resection with 35 patients who had a similar stage of disease but did not undergo surgical exploration [24]. After a follow-up of 12 years, $41 \%$ of patients were cured with surgery and significantly more patients developed LM with conservative treatment (29 vs. $5 \%$; p < 0.001 ). The 15 -year disease-related survival was $98 \%$ after surgery and $74 \%$ after medical treatment $(\mathrm{p}<0.001)$. These results demonstrate that routine surgical exploration increases survival in pa- 


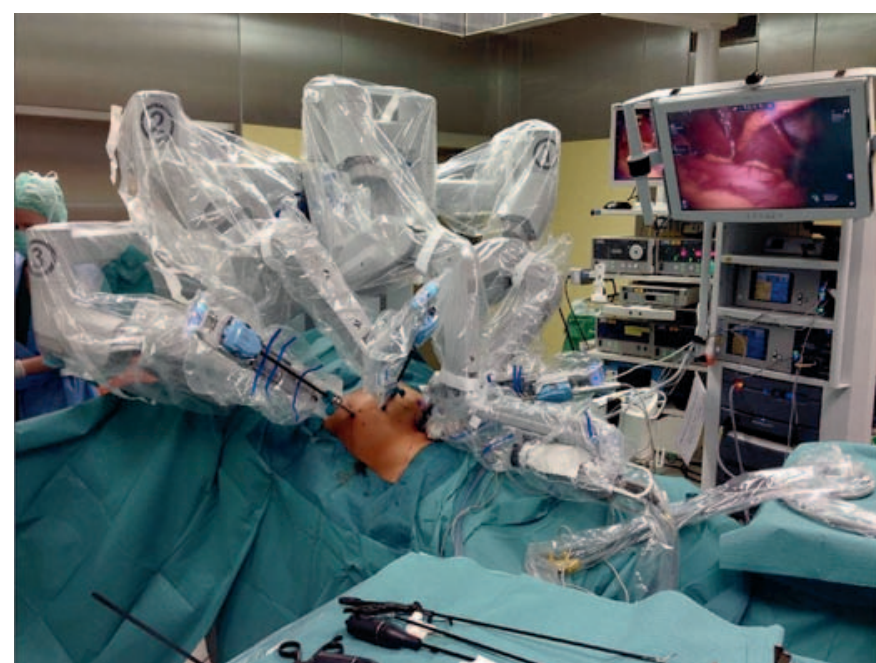

Fig. 3. Robotic-assisted setup for enucleation of a pancreatic neuroendocrine neoplasia.

tients with sporadic ZES by increasing disease-related survival and reducing the rate of advanced disease. Therefore, routine surgical exploration should be performed in all patients with sporadic gastrinomas without evidence of diffuse hepatic metastases and in those who are fit for surgery.

\section{Duodenal Gastrinomas}

DUODX should be routinely performed for all patients with ZES. Recently, Norton et al. [25] underlined the importance of DUODX in patients with ZES. They performed DUODX in 79 patients, and no DUODX was performed in 64 patients. Gastrinoma was found in $98 \%$ with DUODX compared with $76 \%$ with no DUODX. They could show that the use of routine DUODX increases the short- and long-term cure rate. Duodenal exploration is undertaken via longitudinal DUODX in the descending part of the duodenum. Small tumors can be identified by palpation. Duodenal tumors smaller than $5 \mathrm{~mm}$ can be enucleated with the overlying mucosa (fig. 4); larger tumors are excised with full thickness excision of the duodenal wall. After completion of this exploration the DUODX is cautiously sutured longitudinally.

Because of the high incidence of lymph node metastases associated with duodenal gastrinomas, prophylactic lymph node dissection should be performed [1]. Bartsch et al. [26] could demonstrate in 41 patients who underwent potentially curative surgery that systematic lymphadenectomy with excision of more than 10 lymph nodes resulted in a higher rate of biochemical cure after surgery than no or selective lymphadenectomy ( 13 of 13 vs. 18 of 28 patients; $\mathrm{p}=0.017$ ), and a reduced risk of death ( 0 of 13 vs. 7 of 24 patients; $\mathrm{p}=0.037$ ). Another study analyzed the distribution of lymph node metastases found at the time of operation in $38 \mathrm{pa}-$ tients with sporadic duodenal gastrinomas by mapping their location in relation to the duodenal primary [27]. Patients who had primary duodenal tumors located above the ampulla of Vater generally harbored positive lymph nodes in the superior periduodenal area, celiac axis, or periportal area. Those with primary tumors in

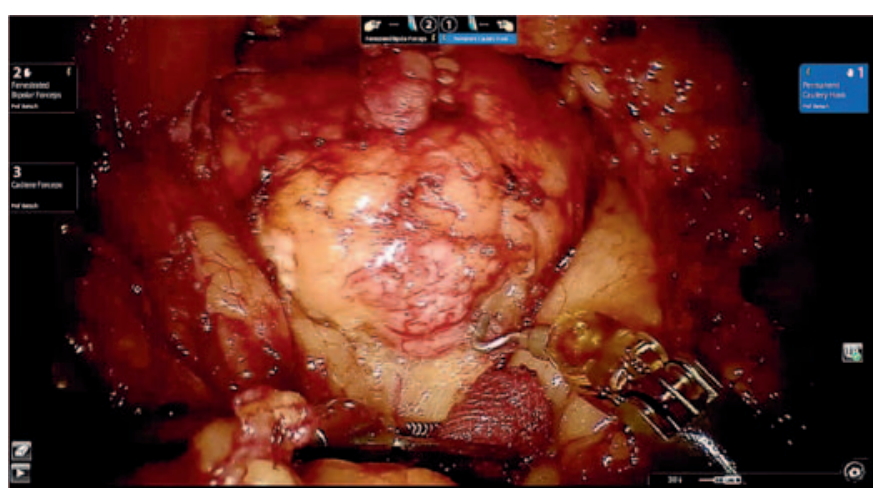

Fig. 4. Robotic-assisted enucleation of a pancreatic neuroendocrine neoplasia.

the third and fourth portions of the duodenum had positive lymph nodes located most commonly in the superior mesenteric artery or inferior periduodenal areas. Lymph nodes were found close to the primary tumor in most cases.

\section{Pancreatic Gastrinomas}

The role of operative exploration in patients with sporadic gastrinomas is relatively well defined. Most of these non-MEN1 gastrinomas are solitary, identifiable at laparotomy, and resectable with simple enucleation. Formal pancreatic resections are typically reserved for patients with local tumor invasion. In practice, this leads to distal pancreatic resection, splenectomy, and peripancreatic lymph node dissection for gastrinomas in the pancreatic body or tail. Most of the pancreatic gastrinomas are located in the head of the gland or uncinate process. An enucleation with peripancreatic lymph node dissection is the procedure of choice in gastrinomas of the pancreatic head. For large pancreatic head gastrinomas a partial PPD is justified [11].

\section{Treatment of Rare Functioning pNENs}

Curative surgery is always recommended whenever feasible after careful symptomatic control of the clinical syndrome; the latter may be achieved by medical or locoregional treatments [1]. Curative surgery should include an oncological pancreatic resection with lymphadenectomy. Laparoscopic resection is generally not recommended because of the need for lymphadenectomy and careful inspection for invasion/metastases $[1,11]$.

\section{Management of Liver Metastases}

LM frequently develop during the natural history of pNENs, with an incident rate of liver involvement between 28 and $78 \%$ [28]. In metastatic pNENs, 5-year survival rates are around 40$60 \%$ [16]. Patients with gastrinoma and no metastatic disease have a 20 -year survival rate of $95 \%$ whilst a 10 -year survival of only $15 \%$ is reported when diffuse metastatic liver disease is present [29]. Whenever possible, R0 resection of LM is still considered as the 
gold standard, although several different options to treat LM have become available in the last years, including biotherapy, chemotherapy, targeted treatments (everolimus, sunitinib), local ablative treatments with transarterial embolization/transarterial chemoembolization, radiofrequency ablation (RFA), irreversible electroporation (IRE) or peptide receptor radionuclide therapy (PRRT), and selective internal radiation therapy (SIRT).

\section{Surgery for Liver Metastases}

The interdisciplinary decision to perform liver surgery is based on a multifactorial assessment, taking into account tumor grading, the presence of extrahepatic metastases, and the presence of symptoms [29]. Only $20-30 \%$ of patients with metastatic disease are suitable for radical intent at presentation. Debulking surgery (R2) in incompletely resectable metastatic disease is not universally accepted, but particularly in symptomatic patients it may improve the quality of life when medical treatment failed [29]. For surgery with curative intent, the European Neuroendocrine Tumor Society (ENETS) has proposed the following criteria: (i) resectable G1-G2 liver disease with acceptable morbidity and less than 5\% mortality, (ii) absence of right heart insufficiency, (iii) absence of unresectable lymph node and extra-abdominal metastases, and (iv) absence of diffuse or unresectable peritoneal carcinomatosis [28]. The overall survival after hepatic resection is $46-86 \%$ at 5 years and $35-79 \%$ at 10 years [16]. In referral centers, the 5 -year survival for hepatic resections of patients with NET (neuroendocrine tumor) LM commonly exceeds $60 \%$. Preselection biases due to better performance status or less advanced disease are likely to influence such differences in favor of the outcomes associated with surgery. Nevertheless, resection is associated with a low mortality rate (0-5\%) and an acceptable morbidity (30\%). Analysis of histopathology specimens revealed that the disease burden in the liver is often underestimated, with almost half the number of LM from NETs being undetectable on preoperative imaging [16].

In the last 20 years, non-surgical novel techniques such as RFA, IRE, SIRT, and PPRT have become available and gained popularity mainly as complementary treatment options. The lack of randomized data makes the comparison with a surgical approach in terms of survival benefit and symptomatic relief difficult.

\section{Liver Transplantation for Metastatic NETs}

In selected cases, liver transplantation (LT) has been used to treat LM from NENs. However, considerable controversy exists due to the absence of adequate available data comparing transplantation for unresectable LM to other treatment modalities [30]. In addition, in Germany, there is a serious lack of donor organs for this indication so that only about $1 \%$ of patients with diffuse metastatic pNEN liver disease will receive a liver transplant. However, LT has been advocated in patients with bilateral unresectable LM that are refractory to other treatment. Only a few multicenter studies and several single-center retrospective studies with a small number of patients are available which evaluate the survival benefits of LT for the treatment of NEN metastases [30]. Recent evidence suggests that the 5-year survival after LT for unresectable LM can be as good as $60-80 \%$ in selected patients. Given an expected survival rate of $20-30 \%$ in untreated cases with diffuse LM, it seems that LT may play a role in highly selected patients [30].

\section{Peritoneal Carcinomatosis}

Peritoneal metastases are considered a serious event in virtually all malignancies and usually lead to a poor prognosis. In NEN, they are the direct cause of death in $40 \%$ of patients [31]. In the largest study so far, which included NENs from different origins, 41 patients underwent cytoreductive peritonectomy with or without hyperthermic intraperitoneal chemotherapy (HIPEC). LM were treated during the same operative procedure in $66 \%$ of the patients. Elias et al. [32] reported an overall survival at 5 and 10 years of 69 and 52\%, respectively. Overall survival was not different between patients treated with or without HIPEC, but the disease-free survival was greater in the HIPEC group $(\mathrm{p}=0.018)$. The authors concluded that resection of peritoneal metastases from a NEN is feasible in most of the patients and seems to increase survival rates, without proving whether the addition of HIPEC had a positive or a negative impact [32].

\section{Conclusions}

pNENs are rare but fascinating tumors. Biochemical diagnosis justifies laparotomy in patients with insulinomas and gastrinomas, even if a tumor is not detected preoperatively. Whereas patients with insulinomas are usually cured, a significant surgical cure rate can also be achieved in patients with gastrinoma. The prognosis of pNENs is much better than that of pancreatic adenocarcinoma, even though patients are frequently diagnosed with metastatic disease. Therefore, an aggressive surgical approach leads to long-term survival even in patients with malignant pNENs. Although longterm cure can only be realized in a small proportion of patients, significant long-term palliation can be achieved.

\section{Disclosure Statement}

The authors do not have a conflict of interest to declare. 


\section{References}

1 Falconi M, Eriksson B, Kaltsas G, Bartsch DK, Capdevila J, Caplin M, Kos-Kudla B, Kwekkeboom D, Rind G, Klöppel G, Reed N, Kianmanesh R, Jensen RT; Vienna Consensus Conference participants: ENETS Consensus Guidelines update for the management of patients with functional pancreatic neuroendocrine tumors and non-functional pancreatic neuroendocrine tumors. Neuroendocrinology 2016;103:153-171.

2 Baumann T, Rottenburger C, Nicolas G, Wild D: Gastroenteropancreatic neuroendocrine tumours (GEPNET) - imaging and staging. Best Pract Res Clin Endocrinol Metab 2016;30:45-57.

3 Mathur A, Gorden P, Libutti SK: Insulinoma. Surg Clin North Am 2009;89:1105-1121.

4 Whipple AO, Frantz VK: Adenomas of the islet cells with hyperinsulinism. Ann Surg 1935;101:1299-1335.

5 Fendrich V, Bartsch DK, Langer P, Zielke A, Rothmund M: Diagnosis and therapy in 40 patients with insulinoma. Dtsch Med Wochenschr 2004;129:941946.

6 Öberg K, Modlin IM, De Herder W, Pavel M, Klimstra D, Frilling A, Metz DC, Heaney A, Kwekkeboom D, Strosberg J, Meyer T, Moss SF, Washington K, Wolin E, Liu E, Goldenring J: Consensus on biomarkers for neuroendocrine tumour disease. Lancet Oncol 2015; 16:e435-446.

7 Zollinger RM, Ellison EH: Primary peptic ulcerations of the jejunum associated with islet cell tumors of the pancreas. Ann Surg 1955;142:709-723.

8 Stabile BE, Morrow DJ, Passaro E Jr: The gastrinoma triangle: operative implications. Am J Surg 1984;147: 25-31.

9 Yu F, Venzon DJ, Serrano J, et al: Prospective study of the clinical course, prognostic factors, causes of death, and survival in patients with long-standing ZollingerEllison syndrome. J Clin Oncol 1999;17:615-630.

10 Chastain MA: The glucagonoma syndrome: a review of its features and discussion of new perspectives. Am J Med Sci 2001;321:306-320.

11 Fendrich V, Waldmann J, Bartsch DK, Langer P: Surgical management of pancreatic endocrine tumors. Nat Rev Clin Oncol 2009;6:419-428.

12 Dedieu A, Rault A, Collet D, Masson B, Sa Cunha A Laparoscopic enucleation of pancreatic neoplasm. Surg Endosc 2011;25:572-576.
13 Zhao YP, Zhan HX, Zhang TP, Cong L, Dai MH, Liao Q, Cai LX: Surgical management of patients with insulinomas: result of 292 cases in a single institution. J Surg Oncol 2011;103:169-174.

14 Mehrabi A, Hafezi M, Arvin J, Esmaeilzadeh M, Garoussi C, Emami G, Kössler-Ebs J, Müller-Stich BP, Büchler MW, Hackert T, Diener MK: A systematic review and meta-analysis of laparoscopic versus open distal pancreatectomy for benign and malignant lesions of the pancreas: it's time to randomize. Surgery 2015; 157:45-55.

15 Carrere N, Abid S, Julio CH, Bloom E, Pradere B: Spleen-preserving distal pancreatectomy with excision of splenic artery and vein: a case-matched comparison with conventional distal pancreatectomy with splenectomy. World J Surg 2007;31:375-382.

16 Tamburrino D, Spoletini G, Partelli S, Muffatti F, Adamenko O, Crippa S, Falconi M: Surgical management of neuroendocrine tumors. Best Pract Res Clin Endocrinol Metab 2016;30:93-102.

17 Partelli S, Cirocchi R, Crippa S, Cardinali L, Fendrich V, Bartsch DK, Falconi M: Systematic review of active surveillance versus surgical management of asymptomatic small non-functioning pancreatic neuroendocrine neoplasms. Br J Surg 2017;104:34-41.

18 Gagner M: Early experience with laparoscopic resecions of islet cell tumors. Surgery 1996;120:1051-1054.

19 Fernández-Cruz L, Molina V, Vallejos R, Jiménez Chavarria E, López-Boado MA, Ferrer J: Outcome after laparoscopic enucleation for non-functional neuroendocrine pancreatic tumours. HPB (Oxford) 2012; 14:171-176.

20 Langer P, Bartsch DK, Fendrich V, Kann PH, Rothmund M, Zielke A: Minimal-invasive operative treatment of organic hyperinsulinism. Dtsch Med Wochen schr 2005; 130:508-513.

21 Lopez CL, Albers MB, Bollmann C, Manoharan J, Waldmann J, Fendrich V, Bartsch DK: Minimally invasive versus open pancreatic surgery in patients with multiple endocrine neoplasia type 1. World J Surg 2016; 40:1729-1736.
22 Eckhardt S, Schicker C, Maurer E, Fendrich V, Bartsch DK: Robotic-assisted approach improves vessel preservation in spleen-preserving distal pancreatectomy. Dig Surg 2016;33:406-413.

23 Zhan Q, Deng XX, Han B, Liu Q, Shen BY, Peng CH Li HW: Robotic-assisted pancreatic resection: a report of 47 cases. Int J Med Robot 2013;9:44-51.

24 Norton JA, Fraker DL, Alexander HR, et al: Surgery increases survival in patients with gastrinoma. Ann Surg 2006;244:410-419.

25 Norton JA, Alexander HR, Fraker DL, et al: Does the use of routine duodenotomy (DUODX) affect rate of cure, development of liver metastases, or survival in patients with Zollinger-Ellison syndrome? Ann Surg 2004;239:617-625; discussion 626

26 Bartsch DK, Waldmann J, Fendrich V, Boninsegna L, Lopez CL, Partelli S, Falconi M: Impact of lymphadenectomy on survival after surgery for sporadic gastrinoma. Br J Surg 2012;99:1234-1240.

27 Zogakis TG, Gibril F, Libutti SK, et al: Managemen and outcome of patients with sporadic gastrinoma arising in the duodenum. Ann Surg 2003;238:42-48.

28 Pavel M, O’Toole D, Costa F, Capdevila J, Gross D, Kianmanesh R, Krenning E, Knigge U, Salazar R, Pape UF, Öberg K; Vienna Consensus Conference participants: ENETS Consensus Guidelines update for the management of distant metastatic disease of intestinal pancreatic, bronchial neuroendocrine neoplasms (NEN) and NEN of unknown primary site. Neuroendocrinology 2016;103:172-185.

29 Frilling A, Clift AK: Therapeutic strategies for neuroendocrine liver metastases. Cancer 2015;121:11721186

30 Vilchez V, Gedaly R: Liver transplantation for the treatment of neuroendocrine liver metastases. Best Pract Res Clin Endocrinol Metab 2016;30:141-147.

31 Elias D, Sideris L, Liberale G, et al: Surgical treatment of peritoneal carcinomatosis from well-differentiated digestive endocrine carcinomas. Surgery 2005; 137: 411-416.

32 Elias D, David A, Sourrouille I, Honoré C, Goéré D, Dumont F, Stoclin A, Baudin E: Neuroendocrine carcinomas: optimal surgery of peritoneal metastases (and associated intra-abdominal metastases). Surgery 2014 155:5-12. 